\title{
Low-loss Hybrid Silicon Tapers
}

\author{
Paolo Pintus ${ }^{1, *}$, Martijn J. R. Heck, Géza Kurczveil and John E. Bowers \\ Department of Electrical and Computer Engineering \\ University of California Santa Barbara \\ Santa Barbara, CA 93106 USA \\ ${ }^{1}$ Permanent address: Scuola Superiore Sant'Anna, Pisa, Italy \\ p.pintus@sssup.it
}

\begin{abstract}
Two types of hybrid silicon tapers are studied. Single taper loss is $0.3-0.5 \mathrm{~dB}$, enabling integration of III/ $\mathrm{V}$ actives on silicon-on-insulator passive circuitry with low loss.

Keywords-Hybrid integration, silicon-on-insulator technology

\section{INT RODUCTION}

Hybrid silicon photonics, where III/V structures are integrated with silicon-on-insulator (SOI) structures by means of wafer bonding, have shown to be very promising. This is highlighted by the recent demonstration of a $50-\mathrm{Gbps}$ highspeed 4-channel integrated wavelength-division multiplexed link, using hybrid silicon lasers [1]. For integration of hybrid silicon devices, such as lasers, amplifiers, modulators and detectors, with passive SOI-based silicon devices, e.g. waveguides or multiplexers, low-loss mode conversion from the silicon waveguide mode to the hybrid mode and vice versa is essential. To achieve this tapered structures can be used $[2,3]$.

In active hybrid structures containing quantum wells (QWs) it is not easy to determine taper losses, since the tapers or a part thereof will be optically pumped and hence the losses become injection current dependent. As a result it is impossible to separate loss contributions from intraband absorption, freecarrier absorption, scattering and from mode conversion. It is however necessary to have a thorough understanding of all these contributions to be able to optimize the taper design.

In this work for the first time we present a detailed study into the linear, passive loss and mode conversion loss contributions of two different types of hybrid silicon to silicon tapers that were previously used by us $[2,3]$. This is done by using a blue-shifted bandgap in the QWs, making them transparent for the characterization wavelength of $1.55 \mu \mathrm{m}$, hence eliminating intraband absorption while retaining all other waveguide properties.

\section{TAPER DESIGN AND REALIZATION}

Hybrid silicon structures are realized by first defining silicon waveguides on an SOI wafer. Waveguides are etched $0.35 \mu \mathrm{m}$ into a $0.70-\mu \mathrm{m}$ silicon layer. The buried oxide layer is $1.0 \mu \mathrm{m}$. Passive silicon waveguide width is $0.8 \mu \mathrm{m}$ to ensure single-transverse mode operation and taper excitation. Hereafter a III/V die with an epitaxial layer design as shown in Fig. 1 and corresponding to [4] is bonded to the SOI. Finally in a three-step etch process the mesas and tapers are etched into the III/V. In this work we have shifted the bandgap of the quantum wells (QWs) to $1.40 \mu \mathrm{m}$, i.e. well below the wavelength of $1.55 \mu \mathrm{m}$ we use for characterization. By doing this we eliminate QW absorption and the need for electrical pumping. This simplifies the process significantly and increases the uniformity and yield. Moreover without the need for electrical probe pads, the structures have a smaller footprint and we can increase the number of test devices on the die. Fig. 1 shows a top metal contact layer, which is used for etch masking to keep the process compatible with and the results representative for the work in e.g. [2-4].

In this work we consider two taper designs, as shown in Fig. 2. In Type 1 tapers the $0.8-\mu \mathrm{m}$ silicon waveguide is tapered out to $2 \mu \mathrm{m}$ where the III/V taper starts. Tapering of the $\mathrm{p}$-InP layer starts almost immediately after the start of the other two taper levels, allowing a $1.2-\mu \mathrm{m}$ margin of the $\mathrm{n}-\mathrm{InP}$ and $\mathrm{SCH}$ levels for fabrication tolerance purposes. This taper has been widely used by us, e.g. in [2]. For our recent work in [3] we have designed a Type 2 taper, where the mode is more gradually converted from the silicon mode to the hybrid mode. In this case the three taper levels start at different positions, requiring a total taper length of $160 \mu \mathrm{m}$. In both taper types the p-InP taper flares out to $4 \mu \mathrm{m}$, after which the wide III/V mesa starts. Mesa width is $24 \mu \mathrm{m}$ for Type 1 and $14 \mu \mathrm{m}$ for Type 2 .

To vary the overlap of the hybrid mode with the QWs, we use two different widths of silicon waveguide in the hybrid section, namely 1.0 and $1.5 \mu \mathrm{m}$. This is realized by tapering the silicon width from $2.0 \mu \mathrm{m}$ down over the length of the III/V taper.

The devices have been realized with a fabrication process as mentioned in [3]. Due to finite lithography resolution the tapers have a tip radius of $\sim 0.5 \mu \mathrm{m}$, as determined by SEM. In Fig. 3 top-view SEM pictures of the realized tapers are shown. As can be seen in Fig. 3(a) the taper start position varies somewhat due to lithography limitations on the narrow stripe (0.5- $\mu \mathrm{m}$ starting width on mask). 


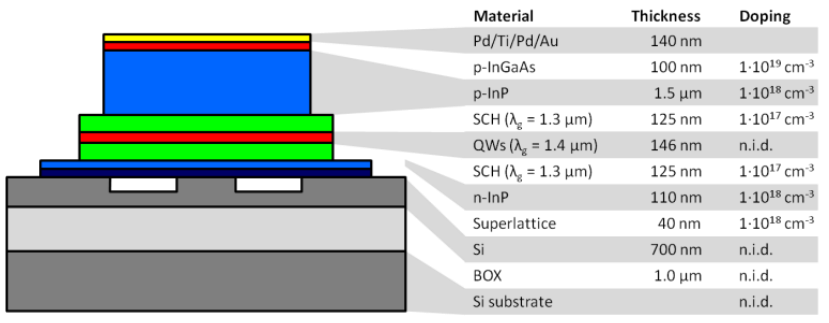

Figure 1. Hybrid silicon cross-section showing the three etch levels and corresponding layer thicknesses and doping levels.
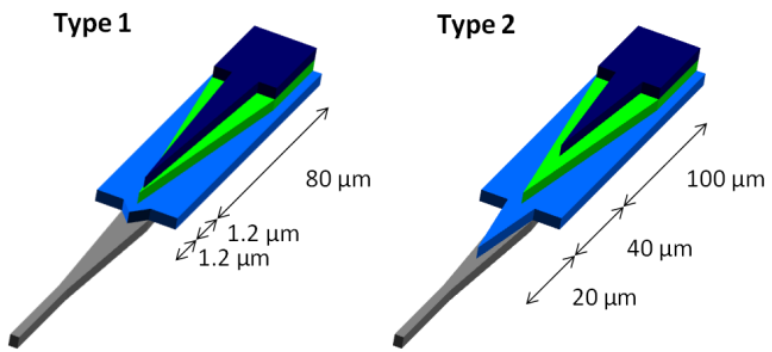

Figure 2. Type 1 and Type 2 tapers showing the silicon waveguide (grey), nInP (light blue), SCH layer cont aining QWs (green) and p-InP top. Taper start offsets are indicated.
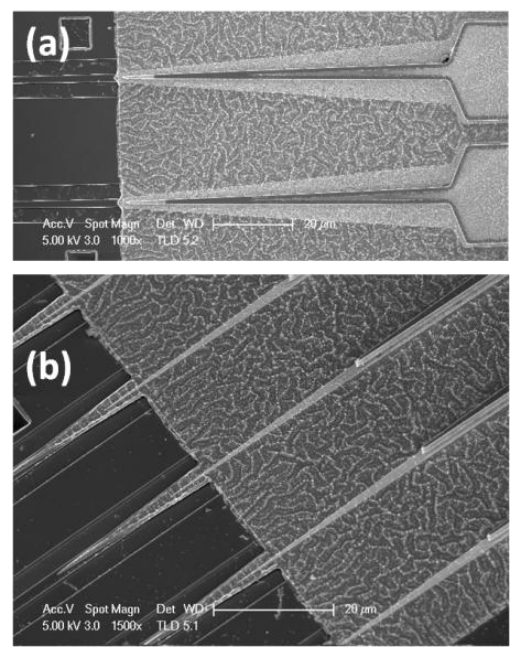

Figure 3. SEM pictures of (a) Type 1 and (b) Type 2 tapers.

\section{EXPERIMENTAL SETUP}

To be able to measure taper losses we have designed test structures as shown in Fig. 4. Hybrid waveguide lengths vary from $200 \mu \mathrm{m}$ to $1800 \mu \mathrm{m}$ and $200-\mu \mathrm{m}$ sections are designed with one, two and three repeats. The silicon waveguides have a $2-\mu \mathrm{m}$ wide polished input and output, angled at $7^{\circ}$, and tapering down to $0.8 \mu \mathrm{m}$. By plotting the insertion losses as a function of hybrid length and repeats, hybrid waveguide propagation losses can be separated from taper losses, as shown in the next section. For increased accuracy these structures have been repeated several times.

The insertion losses are measured using a pair of lensed fibers and the polarization of the input light is controlled to transverse electric (TE).

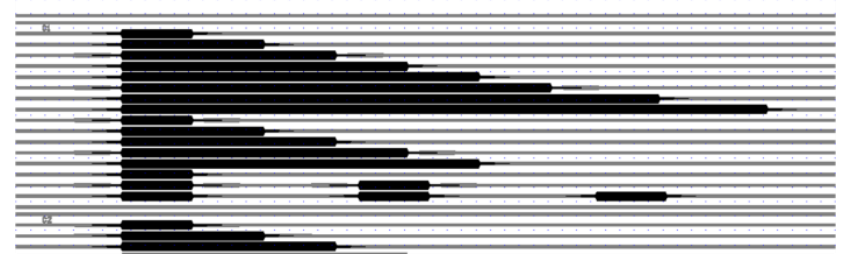

Figure 4. Mask layout showing silicon waveguides (grey) and p-InP etch definition (black). Silicon waveguides have angled inputs and outputs (not shown).

\section{RESULTS}

In Fig. 5(a) the power transmitted through hybrid waveguides with lengths of $200-1800 \mu \mathrm{m}$ is plotted for the two silicon waveguide widths and both Type 1 and 2. A linear fit through the data gives the hybrid waveguide loss. Results are summarized in Table 1. As expected the hybrid sections with $1.0-\mu \mathrm{m}$ silicon waveguides show $17-20 \mathrm{~dB} / \mathrm{mm}$ higher losses than the $1.5-\mu \mathrm{m}$ waveguides. This can be explained by the fact that the optical mode is pushed more into the doped and higher-loss III/V when the silicon is narrowed. We explain the difference between Type 1 and Type $21.0-\mu \mathrm{m}$ results by assuming that the narrower Type 2 mesa confines higher order modes better and hence decreases radiation loss of unguided modes.

The offset in Fig. 5(a) gives the loss of a pair of tapers, when compared to a passive (no hybrid) silicon waveguide. These are given in Table 1. The taper losses can also be compared by taking the slope of the plot in Fig. 4(b), where loss as a function of the number of $200-\mu \mathrm{m}$ hybrid sections in a waveguide is plotted. By subtracting the hybrid waveguide propagation losses, as determined by Fig. 5(a), the loss of a pair of tapers can be determined. These results also given in Table 1.

When comparing Type 1 and 2 taper losses it can be seen that Type 1 losses are around $0.5 \mathrm{~dB}$ and Type 2 losses are $0.3 \mathrm{~dB}$, i.e. significantly lower. The reas on is that Type 2 tapers more gradually convert the silicon mode to the hybrid mode and vice versa. Another important observation is that tapers to $1.0-\mu \mathrm{m}$ waveguides have higher loss than to $1.5-\mu \mathrm{m}$ waveguides. This can be understood as the mode in the $1.5-\mu \mathrm{m}$ hybrid section has a higher overlap with the silicon than in the $1.0-\mu \mathrm{m}$ case. Hence the center of gravity of the mode does not have to be shifted up and down as much, thereby decreasing conversion losses. Another contribution is the higher overlap with the higher-loss III/V layers, as explained above. Finally due to the lower overlap with the III/V layers, the $1.5-\mu \mathrm{m}$ case is more robust to misalign ment and fabrication errors.

It is important to note that strictly speaking the presented technique only gives us the loss per taper pair. Potentially the taper can excite multiple transverse modes in the hybrid waveguide, which will have different propagation constants and loss values. Hence the propagation losses from (singlemode) $0.8-\mu \mathrm{m}$ silicon waveguides to the hybrid section might differ from those in the vice versa direction. More specific these losses will then also be wavelength and hybrid waveguide-length dependent. This is currently under investigation. 

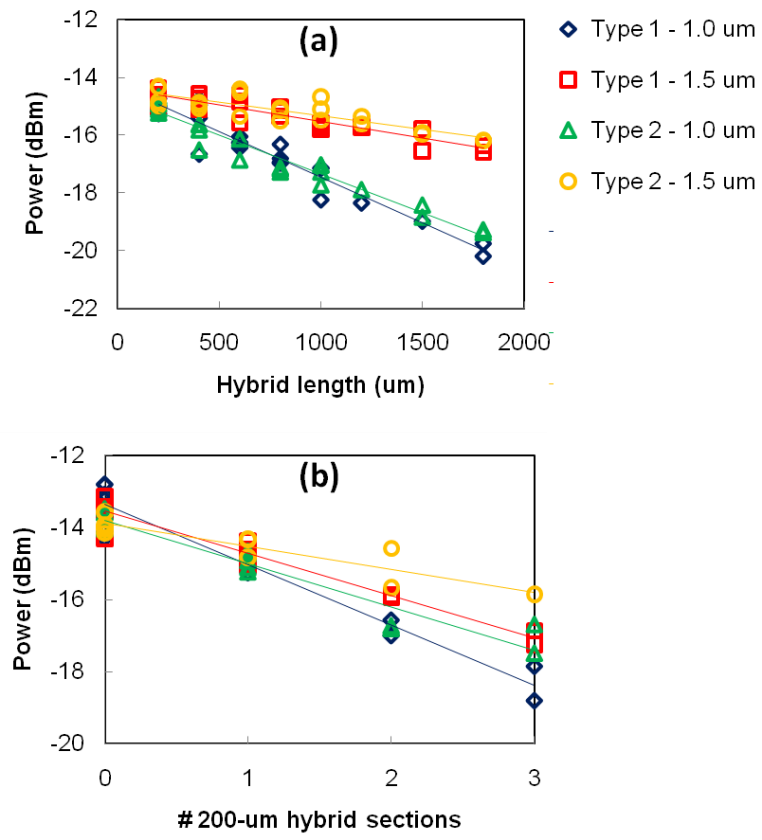

Figure 5. Measured in-fiber output power after propagation through structures with (a) various hybrid waveguide lengths and (b) various number of $200-\mu \mathrm{m}$ hybrid sections.

TABLE I. EXPERIMENTAL LOSS VALUES

\begin{tabular}{|lccr|}
\hline & $\begin{array}{c}\text { Hybrid loss } \\
(\mathbf{d B} / \mathbf{m m})\end{array}$ & ${\text { Taper loss }(\mathbf{d B})^{\mathbf{a}}}$ & Taper loss (dB) $^{\mathbf{b}}$ \\
\hline \hline Type $1-1.0 \mu \mathrm{m}$ & $3.2 \pm 0.2$ & $0.52 \pm 0.05$ & $0.47 \pm 0.11$ \\
Type $1-1.5 \mu \mathrm{m}$ & $1.2 \pm 0.1$ & $0.47 \pm 0.04$ & $0.42 \pm 0.07$ \\
Type $2-1.0 \mu \mathrm{m}$ & $2.7 \pm 0.1$ & $0.33 \pm 0.05$ & $0.43 \pm 0.07$ \\
Type $2-1.5 \mu \mathrm{m}$ & $1.0 \pm 0.1$ & $0.22 \pm 0.05$ & $0.25 \pm 0.08$ \\
\hline & & a. & As determined from the slope of Fig. 5(b) \\
& & b. $\quad$ As determined from the offset of Fig. 5(a)
\end{tabular}

\section{CONCLUSION}

By eliminating intraband absorption effects, passive propagation losses, including mode-conversion losses in hybrid silicon tapers have been quantified. The tapers presented show losses of 0.5 and $0.3 \mathrm{~dB}$ per taper transition for the two taper designs presented, i.e. they add 1.0 to $0.6 \mathrm{~dB}$ to the insertion loss of hybrid silicon devices integrated on passive SOI integrated circuits. These losses are significantly lower than the 1-2 dB coupling loss values that are generally obtained in other hybrid integration approaches [5], showing the clear advantage of our integration approach.

Although our Type 2 tapers show lower losses than Type 1 tapers, it is not evident yet which type is most advantageous in active hybrid devices, i.e. having QWs with a bandgap corresponding to the operating wavelength. Electrical current is generally injected through the top $\mathrm{p}$-contact, i.e. the top $\mathrm{p}$-InP taper. If the QWs are in the second $(\mathrm{SCH})$ taper level, the overlap between the p-InP taper and the SCH taper should be maximized to avoid unpumped, i.e. absorbing, regions in the taper. It can be seen from Fig. 2 that Type 1 tapers offer a clear advantage over Type 2 tapers in this respect. This remains the object of further study. These results are widely applicable to hybrid silicon photonic integrated circuits, and apply to hybrid silicon lasers, modulators, amplifiers and photodetectors.

\section{ACKNOWLEDGMENT}

We thank Jon Peters for fabricating the chips and Jag Shah for supporting this research through DARPA LASOR contract W911NF-04-9-0001.

\section{REFERENCES}

[1] A. Alduino et al., "Demonstration of a High Speed 4-Channel Integrated Silicon Photonics WDM Link with Hybrid Silicon Lasers" in Int egrated Photonics Research, Silicon and Nanophotonics, OSA Technical Digest (CD) (Optical Society of America, 2010), paper P DIWI5.

[2] H. Park, Y.-H. Kuo, A.W. Fang, R. Jones, O. Cohen, M.J. Paniccia and J.E. Bowers, "A hybrid AlGaInAs-silicon evanescent preamplifier and photodetector," Opt ics Express, vol. 15, no. 21, pp. 13539-13546, 2007

[3] G. Kurczveil, M.J.R. Heck, J.D. Peters, J.M. Garcia, D. Spencer and J.E. Bowers, "An Integrated Hybrid Silicon Multiwavelength AWG Laser," IEEE Journ. of Sel. Top. in Quant. Electron., to be published, doi: 10.1109/JST QE.2011.2112639

[4] H. Park, A. W. Fang, O. Cohen, R. Jones, M.J. Paniccia, and J.E. Bowers, "An electrically pumped AlGaInAs-Silicon Evanescent Amplifier," IEEE Photon. Technol. Lett. 19, 230-232 (2007).

[5] A. Poustie, "Hybrid integration for advanced photonic devices," in Proc. of SPIE, vol. 7135, 713502, 2008, doi: 10.1117/12.803089 